\title{
Evidence-based decision-making within the context of globalization: A "Why-What-How" for leaders and managers of health care organizations
}

\author{
Véronique Lapaige \\ Canadian Health Services Research \\ Foundation Fellow; PRO-ACTIVE \\ Research Program (Participatory \\ and Evaluative Research Program to \\ Optimize Workplace Management: \\ Application of Knowledge, Transfer of \\ Expertise, Innovative Interventions, \\ Training Transformational Leaders) \\ Pavillon Ferdinand-Vandry, CIFSS \\ (Centre intégré de formation en \\ sciences de la santé), Laval University, \\ Quebec City, Quebec Canada
}

\begin{abstract}
In the globalized knowledge economy, the challenge of translating knowledge into policy and practice is universal. At the dawn of the 21 st century, the clinicians, leaders, and managers of health care organizations are increasingly required to bridge the research-practice gap. A shift from moving evidence to solving problems is due. However, despite a vast literature on the burgeoning field of knowledge translation research, the "evidence-based" issue remains for many health care professionals a day-to-day debate leading to unresolved questions. On one hand, many clinicians still resist to the implementation of evidence-based clinical practice, asking themselves why their current practice should be changed or expanded. On the other hand, many leaders and managers of health care organizations are searching how to keep pace with the demand of actionable knowledge. For example, they are wondering: (a) if managerial and policy innovations are subjected to the same evidentiary standards as clinical innovations, and (b) how they can adapt the scope of evidence-based medicine to the culture, context, and content of health policy and management. This paper focuses on evidence-based health care management within the context of contemporary globalization. In this paper, our heuristic hypothesis is that decision-making process related changes within clinical/managerial/policy environments must be given a socio-historical backdrop. We argue that the relationship between research on the transfer of knowledge and its uptake by clinical, managerial and policy target audiences has undergone a shift, resulting in increasing pressures in health care for intense researcher-practitioner collaboration and the development of "integrative KT platforms" at the crossroads of different fields (the field of knowledge management and the field of knowledge translation). The objectives of this paper are: (a) to provide an answer to the questions that health professionals ask most frequently about "Why" and "How" to bridge the know-do gap, (b) to illustrate by a Canadian example how the PRO-ACTIVE program helps in closing the evidence-based practice gap.
\end{abstract}

Keywords: globalization, knowledge, know-do gap, research-practice gap, knowledge translation, knowledge sharing, evidence-based decision-making, evidence-based medicine, evidencebased health care management

\section{Introduction}

In today's era of globalization, the challenge of translating knowledge into policy and practice is universal. ${ }^{1}$ At the dawn of the 21 st century, bridging the research-practice gap is one of the most important challenges for public health, and all health care professionals are increasingly required to bridge this know-do gap. ${ }^{2-8}$ A shift from moving evidence to solving problems is due. However, many health care professionals at clinical, organizational, and policy-making levels are making haphazard attempts to render their practices more congruent with the knowledge society and the changing care environment in which they work. Despite a vast literature on the burgeoning field of knowledge translation research, the "evidence-based" issue remains for them 
a day-to-day debate leading to unresolved questions. On one hand, many clinicians still resist to the implementation of evidence-based clinical practice, asking themselves why their current practice should be changed or expanded..$^{9-13}$ On the other hand, many leaders and managers of health care organizations are searching how to keep pace with the demand of actionable knowledge. For example, they are wondering: "Why and how has my professional practice changed or expanded? Is evidence-based managerial practice akin to evidence-based medicine? Should I adjust to the social and historical context of the knowledge economy, or should this be responsibility of my health care institution, or even my management team? How can I stay current with developments in evidence-based or knowledge-informed practice, ie, in such an environment, how can I become a knowledgeable manager or an evidence-based decision-maker?"*

In this paper, we present a reasoned response to the questions that Canadian health care managers ask most frequently about the specifics of a knowledge-translation strategy fine-tuned to the type of decisions their face and the type of decision-making environment in which they work.

This paper focuses on evidence-based health care management within the context of contemporary globalization. It is grounded on the results of an integrated " $K T$ research" ("integrated knowledge translation research") whose purpose was to develop a $K T$ learning platform for Canadian health care managers, and which is part of the PRO-ACTIVE research program.

In this paper, we provide the reader a more comprehensive account of globalization's transformative power of public health/health care decision-making environments. The paper highlights the relationships between the evidence-based decision-making movement and the sociohistorical process of globalization. We also offer a reference framework for an in-depth understanding about the why and the how to bridge the know-do gap in managerial decision-making environment.

The paper aims: (a) to provide an answer to the questions that health care professionals ask most frequently about " $W h y$ " and "How" to bridge the know-do gap, (b) to illustrate by a Canadian example how the PRO-ACTIVE research program helped in closing the evidence-based managerial practice gap.

\footnotetext{
*These issues, raised by Canadian health care managers, were shared with the author by Mr. Fortin, Sector Coordinator of Knowledge Management at the Information and Knowledge Management Branch of the Montérégie Regional Health and Social Services (Personal communication, May 2nd, 2008).
}

This paper is structured into two sections. The first section presents the development of the evidence-based practice movement. We describe the principles, the origins, and the five-step process of evidence-based medicine. The second section is organized around the "Why-What-How" triptych. To answer the question "Why," we situate the evidence-based decision-making movement in its socio- historical context and shed light on the need for target audiences (clinicians, managers, public policy-makers) to develop evidence-based decision-making competencies. We then address the "What" question, which focuses on evidence-based decision-making and requires a distinction between the roles of clinical practice and managerial within the evidence-based health care movement. We also draw on the "What" question to describe the different types of knowledge in the health sector and explain the corresponding forms of management as they relate to specific clinical or managerial levels of the professional practice. Finally, we provide an answer to the "How" question with proposals on how to foster integrated learning readiness at the individual, team (group) and organizational levels. These proposals are accompanied by a reference framework that should prove useful to managers, and an illustration of a knowledge-strategy fine-tuned to managerial practice is provided with the example of the PRO-ACTIVE research program.

\section{Evidence-based decision-making movement: origins, principles, and five-step process of evidence-based medicine}

The evidence-based decision-making movement can be traced back to the early 1990s, since then the process of drawing together, analyzing, and synthesizing evidence from the best available research has become a central practice across many areas of administration. ${ }^{4}$

\section{Definitional evolution of evidence-based medicine}

Evidence-based medicine (EBM) has its roots in clinical epidemiology, owing much to Cochrane's critique of the effectiveness and efficiency of health services. Cochrane ${ }^{14}$ argued that commonly used treatments and investigations in health care systems have not been shown to be effective in terms of clear and convincing evidence. A growing literature on geographical variations has also underlined how professionals' ways of managing similar health problems are diverse and how great is the gap between scientific evidence and clinical practice. 
The EBM model has its origin in the Department of Clinical Epidemiology and Biostatistics at McMaster University in Canada. In 1981, members of that department published in the Canadian Medical Association Journal a series of articles that were intended to teach clinicians how to critically appraise the medical literature. In 1990, they began to move beyond teaching critical appraisal skills into developing a new philosophy of medical education labelled EBM. In this new model, physicians relied heavily on the medical research literature, rather than on textbooks or tradition when approaching patient care problems. This perception of EBM was similar to Cochrane's early works on efficiency and effectiveness, as it focused on the outcomes of a given action or intervention.

However, the uniqueness of McMaster's work was the introduction of the "process" of EBM. The key stages to a practice using evidence base began to emerge:

1. A problem is given to the students in the form of a case study.

2. The students are required to search the evidence associated with the case study.

3. The evidence is applied to the case study.

4. Effectiveness evaluation of their intervention is undertaken associated with the outcome of the case study.

This confirms that evidence-based clinical practice involves the execution of a series of steps in providing care, treatment or intervention and in evaluating the effectiveness of it. Sackett, often considered as the father of EBM, has therefore defined evidence-based medicine as "the conscientious, explicit and judicious best evidence in making decisions about the care of individual patients" (p 71). ${ }^{4} \mathrm{EBM}$ is also the application of knowledge of medical informatics and clinical epidemiology to the treatment of individual patients as it involves the integration of best research evidence with clinical expertise and patient values. ${ }^{4} \mathrm{EBM}$ has been described partly as a philosophy, partly a skill, and partly as the application a set of tools.

However, in 2008, many health care professionals remain unfamiliar with the methods and the "philosophy" of evidence-based medicine. The concern of many health care professionals is either that evidence-based medicine constitutes a "cookbook" medicine or that it may lead to a prescriptive practice where only one way - and often the cheapest - is recommended for providing care. Nevertheless, different authors disagree with the "cookbook" notion by suggesting that evidence-based medicine is the integration of scientific and experiential knowledge into clinical practice. ${ }^{3-4,15}$

\section{Evidence-based medicine: Role and five-step approach}

Depending on author's perspective, EBM is employed to serve different purposes. ${ }^{3,4,16}$ Stated goals of EBM include the following: (a) to enhance the quality of care by providing clinicians with information on which to base their clinical decisions; (b) to ensure that individual patient care is based on the most up-to-date evidence and results in the best possible outcomes; (c) to encourage physicians to maximize the likelihood of positive outcomes over many patients rather than just the patient at hand; (d) to minimize the gap between research and practice. The Institute of Medicine pointed out three categories of problems related to this researchpractice gap: the overuse of some health care interventions (particularly in circumstances where they are not effective); the underuse of interventions (that are proven to be effective but are not applied appropriately); and the misuse of interventions (particularly when the evidence of effectiveness is unclear and leads to wide variations of practice). ${ }^{16}$

The practice of EBM is generally described as having five well-defined steps:

1. Formulate a structured, clear, and answerable clinical question from a patient's problem or information need.

2. Search the literature for relevant clinical articles that might answer the question.

3. Conduct a critical appraisal on the selected research articles and rank the evidence for its validity and usefulness (clinical application).

4. Formulate and apply a clinical intervention based on the useful findings, or best "evidence."

5. Evaluate the clinician's performance. ${ }^{4}$

Specific competencies of health care professionals are required particularly for the first and the second steps which are identified as the most commonly taught aspect of EBM. Inherent in the definition and practice of EBM is in fact the ability to find the best available evidence from research. ${ }^{15}$

\section{Evidence-based medicine: Type of knowledge and hierarchy of evidence}

The evidence-based medicine "movement" - evidencebased practice, evidence-based health care, evidence-based health policy, and other concepts with "evidence-based"prefix - emerged in the early 1990s and quickly evolved into more general calls for the adoption of an evidencebased decision-making culture at all levels of the health care system. ${ }^{5}$ Evidence-based medicine movement's advocates want patients, health professionals, health care managers, ${ }^{6}$ 
and public policymakers ${ }^{7}$ to pay attention to the best findings from health care research that meet the dual requirements of being both scientifically valid and ready for application. Over the last decade, the "evidence-based" concept has even spread the sectors outside health care, with the development of evidence-based social work, evidence-based criminal justice, and evidence-based education. ${ }^{8}$ However, despite the diffusion and adoption of the ideas associated with evidence-based health care, evidence-based practice is not without controversies. ${ }^{17-22}$ Such controversies have centred primarily on the conception of evidence..$^{23-27}$

In terms of evidence in health care, the methodological principles which point towards the highest level of evidence are scientific principles, and the paradigm of scientific knowledge belongs to the natural sciences. ${ }^{18}$ In the evidence-based medicine/evidence-based decision-making movement, evidence is therefore scientific evidence and fundamentally, science is experimental, ${ }^{18}$ and science is interpreted as quantitative in its methods and results. Evidence is also research-based evidence from the empirico-analytical paradigm. ${ }^{3}$ Evidence-based medicine favors secondary research, and a key strategy of secondary research is the systematic review. ${ }^{3}$ Even if evidence-based medicine's advocates have recognized that scientific evidence is narrow and needs to be integrated with a more expansive range of evidentiary sources, they still focus on the use of the "best" sources of evidence. ${ }^{4,28-29}$

Muir $\mathrm{Gray}^{3}$ identifies the various types of research evidence into a hierarchy of type and the strength upon which clinical practice can be based, the best evidence being produced by experimental research in which sources of bias and confounding variables are controlled. Different hierarchies of evidence and classification criteria based on the study design and its methodological rigor were successively developed by the Canadian Task Force on the Periodic Health Examination in 1994, the US Preventive Services Task Force in 1996, and the Oxford Centre for Evidence-Based Medicine in 2001. ${ }^{30-32}$

EBM thus privileges formal, publicly, assessable propositional knowledge arising through basic research. In evidence-based health care, there is a thus strong emphasis on evidence from a quantitative or scientific research base to support practice as opposed to the value of softer or more qualitative approaches. Based on certain scientific standards for assessing the quality of research evidence, a hierarchy of research designs is now well established. At the top of this hierarchy of evidence is the randomized control trial, which is universally regarded as the gold standard because other research designs fail to control common threats to internal validity (testing, instrumentation, statistical regression, selection biases, and Hawthorne effect).

However, even tough it is well established that individual studies can lead to a different conclusion than systematic reviews, ${ }^{33}$ Weiss ${ }^{34}$ has emphasized the importance of "ideas," not "data," which most profoundly influence managerial and policy-decision making processes (for example, public policy-makers rarely use a regression coefficient to help them a problem). Moreover, public health interventions face "structural impediments" (including practical, political, economic and other constraints) which frequently make rigorous testing community interventions in randomized control trials virtually impossible. ${ }^{35}$ One of the significant challenges confronting research on community interventions is that in contrast to experimental studies, communities are open dynamic systems with a large number of factors that may influence health outcomes. Another major challenge facing evidence-based public health is that its interventions often involve policy changes, and randomly assigning communities is politically impossible. Similarly, the feasibility of using randomized control trials in public heath becomes even more questionable if there is a speculation about potential adverse effects. ${ }^{36}$

\section{Evidence-based decision-making: "Why-What-How" answers for health care managers}

In today's health care systems, everyone makes decisions, from medical specialist to staff manager, and everyone is facing the challenging issue of knowledge-sharing and evidence-based decision-making.

\section{"Why?"}

Why are the concepts of knowledge and evidence now receiving so much attention in health care organizations, research centers, and health funding organizations? Why have they become so fashionable, to the point that the situation sometimes resembles an "evidence-based decision-making's courtship" in today's health care systems?

Since the end of the 1980s, the Canadian health care system (like other health care systems in the so-called developed countries) has been experiencing a major structural crisis that has only deepened over time. As the media point out on a daily basis, this crisis is often attributed to aging populations, budget cuts at the national level, increased emphasis on accountability and transparency, growing complexities of biomedical research, and/or great advances in knowledge and technologies. At the same time, Canada, in an attempt 
to meet efficiency and health equity objectives, has been promoting a knowledge management culture and the creation of an integrated capacity for analysis and decision-making in order to maintain and enhance health among the Canadian public. ${ }^{37-39}$ A macro-analysis has revealed that the crisis in the Canadian health care system is part of a much larger, trans-national problem: the whole public health field is in crisis. This crisis has a close and complex relationship with the social and historical process of globalization, to the point that we can speak of "globalized public health." ${ }^{40}$ Research, knowledge and action in public health are being radically transformed in ways that are closely linked to the key logics of globalization. Public health institutions and health care organizations are facing significant challenges due to changes taking place in the global social environment. This global shift is necessitating a focus on intense researcher-practitioner collaboration in order to develop "integrative KT platforms" at the crossroads of different interdisciplinary fields (the field of knowledge management and the field of knowledge translation and research on knowledge translation). ${ }^{40}$

For two long decades, contemporary globalization ${ }^{\ddagger}$ has been affecting fields as diverse as culture and politics, production, work, finance, education and health. It is also linked to the emergence of new risks to the environment and health, and is undermining the sovereignty of nations as well as the logic

Globalization adheres to the six following logics: (1) the market logic (which advocates that everything is negotiable); (2) the productive logic (which underlies the organizational changes occurring in the new global world, such as lean production and total quality); (3) the cyber logic (related to the ICTs' revolution); (4) the dromocratic logic (from the Greek dromos, for "acceleration," in order to characterize new ways of expressing human relationships in an era of emergency, immediacy, instantaneousness, and speed, where acceleration is the common denominator that unites the other forms); (5) the epidemic logic (which refers to the elimination of frameworks and boundaries and stems from a contagion logic of how knowledge propagates through contact, and which conveys both beneficial and destructive elements, such as viruses, the deregulation of foreign-exchange markets, the rise of religious extremism, and the SARS crisis); and (6) the technoscientific logic (which refers to the alliance between science and technology for increased performance, inseparable from high-performance culture and linked to speed and obeying market laws. This may be seen as a knowledge production logic in which technical know-how tends to guide or trump theoretical knowledge). By adopting these different logics, the new globalized world builds or "governs," as it may be, many societies: the market society, the techno-scientific society, the risk society, the networked and timeless society, and the knowledge society (which is still referred to as the learning society).

¥Although linked to a political and economic movement originating in the 16th century, today's globalization, which emerged in the early 1980s, is fundamentally distinct from the three previous phases of globalization-mercantilist (1498-1763), capitalist (1763-1883), and industrial (1883-1980)-because of different organizational features (the HIV/AIDS pandemic, the H5N1 epidemic, greater inequities, market volatility, money laundering, mass terrorism, climate change, etc.). of the providential state. Every so-called developed country becomes a fully global society, thereby adopting the multiple facets of a risk society, a market society, a techno-scientific society, a networked and timeless society (often referred to as the information society), and a knowledge society, which we prefer to call the cognitive or learning-ready society. ${ }^{40}$ Globalization, which is linked to the development of information and communication technologies (ICTs), works through its various logics by programming social change towards an expansion of the space-time provided for learning readiness. Over the past 25 years there has in fact been a profound transfer of capital toward the intangible or immaterial, and the globalized knowledge economy raises the unavoidable issue of how to manage the immaterial. The acquisition of knowledge and new skills has become a major lever for transforming and developing individuals and their professions - even professional entities - as well as organizations. In the era of contemporary globalization, the learning function has become strategic at all levels of social organization. The world order is moving towards a global knowledge economy in which the success of individuals and organizations, professions, regions and nations will above all be a reflection of their capacity to learn. ${ }^{41}$

At the dawn of the 21 st century, knowledge-sharing is a major issue of the emerging field of "globalized public health" which promotes the development of local/global evidence-based decision-making strategies at the crossroads of the knowledge management model and the burgeoning field of knowledge translation. In today's health care systems, fostering a knowledge-sharing attitude and evidence-based decision-making competency are vital at all levels. Both knowledge generation and knowledge sharing take place at the individual, group and organizational level. Lavis ${ }^{37}$ makes clear different "target audiences" for a body of research knowledge. In our view, various interrelated "target environments of evidence-based decision-making" and/or "target health care levels for an actionable evidence-based message" can be differentiated in order to develop specific knowledge-sharing strategies and activities:

1. The evidence-based decision-making at the micro level including:

a. Evidence-based medicine,

b. Evidence-based practice covering all the clinical health sciences (eg, evidence-based nursing);

${ }^{\S}$ Learning readiness refers to a characteristic openness to knowledge and knowledge development, so it differs from learning per se, which refers to the act of learning. 
2. The evidence-based decision-making at the meso level including:

a. Evidence-based health care management;

3. The evidence-based decision-making at the meso and/or macro level including:

a. Evidence-based public health,

b. Evidence-based health promotion (which relate more specifically to the public health practitioners as well as decision-makers confronted with the development of public policies and program evaluations);

4. The evidence-based decision-making at the macro level including:

a. Evidence-informed policy;

b. The need to create evidence-based health care organizations and to develop evidence-based health care systems.

As a result of the evidence-based decision-making movement, managers, nurses, paramedics, physicians, rehabilitation specialists, public health practitioners and health professionals can no longer be "of their time" (today's era of globalization) without knowing and learning, which ultimately requires further knowledge and the development of knowledge-sharing competencies. Fostering a knowledgesharing attitude and competency of patient care processes are vital for any professional in health care, and integrating evidence-based competencies must occur at both the micro, meso, and macro levels (at the individual, management team or group, and institutional levels) (see Figure 1).

\section{"What?"}

What dimensions of knowledge and what types of evidence do health care managers and other health professionals need? What level of competencies is specifically required?

According to the classic definition provided by Plato ${ }^{42}$ and Locke, ${ }^{43}$ knowledge reflects a belief that must be true and verifiable. However, there is still no universal definition of knowledge. In this article, we use two definitions, one provided by Ballay ${ }^{44}$ and another by Huber ${ }^{45}$ and Nonaka. ${ }^{46}$ Ballay ${ }^{44}$ proposes conceiving knowledge as the integration of "phenomena such as language, memory, learning, experience,

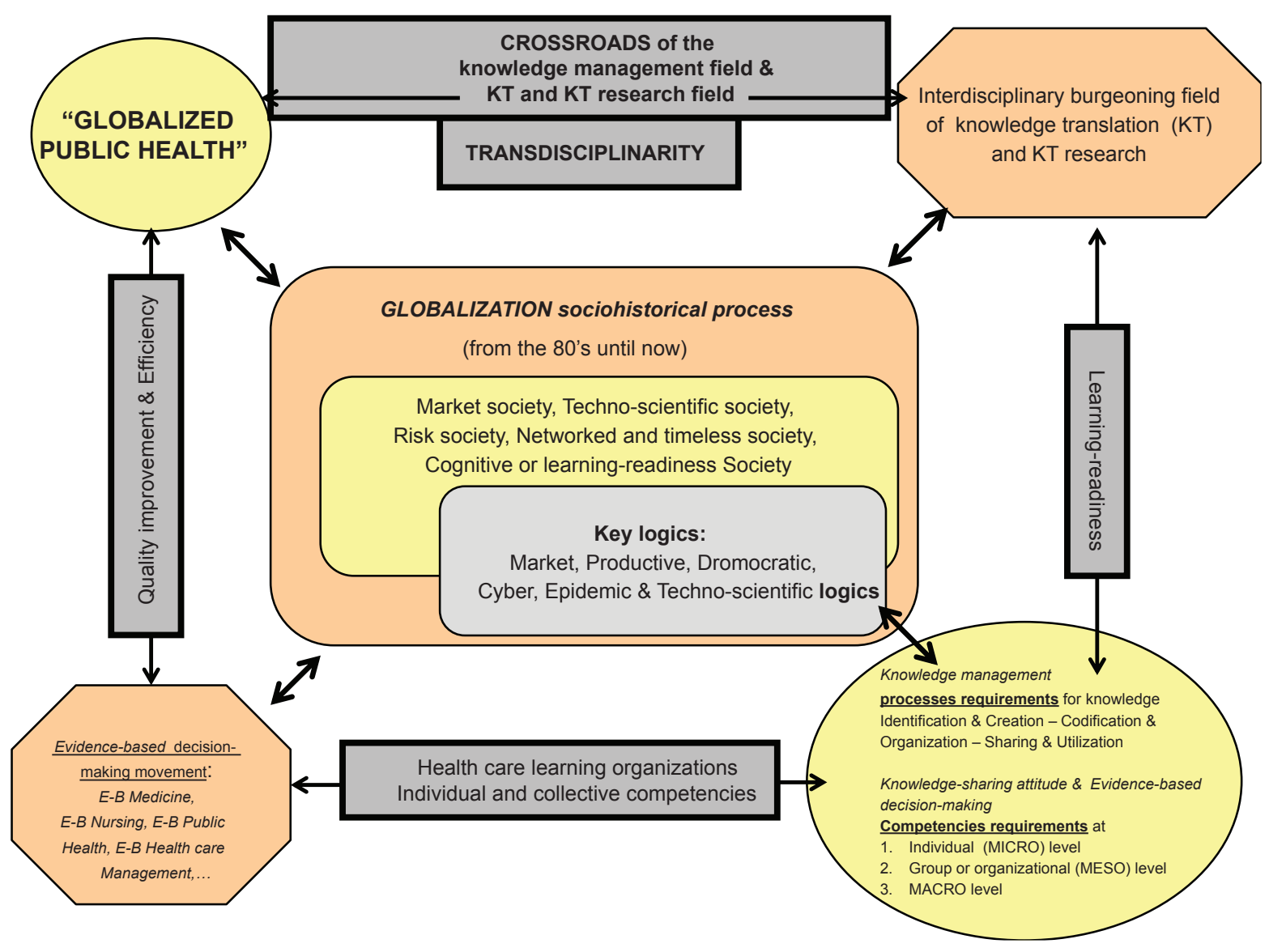

Figure I The "why" answer to evidence-based decision-making. 
perceptions and emotions. [It is] what is present in one's mind, consciously or unconsciously, we are when placed in a situation that requires acting, speaking, learning, experiencing, interpreting, and deciding" (p. 21). On the other hand, Huber ${ }^{45}$ and Nonaka ${ }^{46}$ associate knowledge with behavior or a required act. They understand knowledge as related to a justified belief that enhances the capacity of an entity to act effectively. What we shall retain from these definitions is their comprehensive nature, oriented towards the action of knowledge (its actionable character) and its agentive nature, meaning that knowledge must be initiated by an individual or a group agent.

There are also different dimensions around which knowledge can be characterized such as a storage media, accessibility, typology and hierarchy. ${ }^{46-49}$ These characterizations highlight the conditions under which different knowledge-sharing activities are practised. We will now expand on each of these dimensions.

First, the knowledge storage media may be: (a) the human mind (knowledge is held or internalized by a person, becoming part of their cognitive capital), (b) an institution or organization, (c) technical, physical, or technological storage media (eg, the knowledge found in data banks and reproduced as documents or knowledge residing in computer memory). Knowledge in mind is difficult to access; organizational knowledge is sometimes dispersed whereas document or computer knowledge can be formalized, sharable, and often well structured. In order to effectively develop knowledge-sharing strategies, it is important to pay attention to the most useful storage media at the considered decisionmaking level.

Second, in terms of knowledge hierarchy, different authors draw distinctions between: data, information (evidence) and knowledge (see Figure 2). Data or raw data are a collection of symbols which are devoid of meaning. These aspects of reality have not been put into words (eg, facts, events, images or sound) and are subjected to minimal intervention initiated by a human medium (the human mind which is often backed by a technical or technological medium; then, they transformed into information). Information refers to formatted, filtered and summarized data. Information can be false information or can become evidence. "True information" or "information" may itself be divided into three types: absolute scientific information (which can become absolute researchbased evidence), context-based scientific information

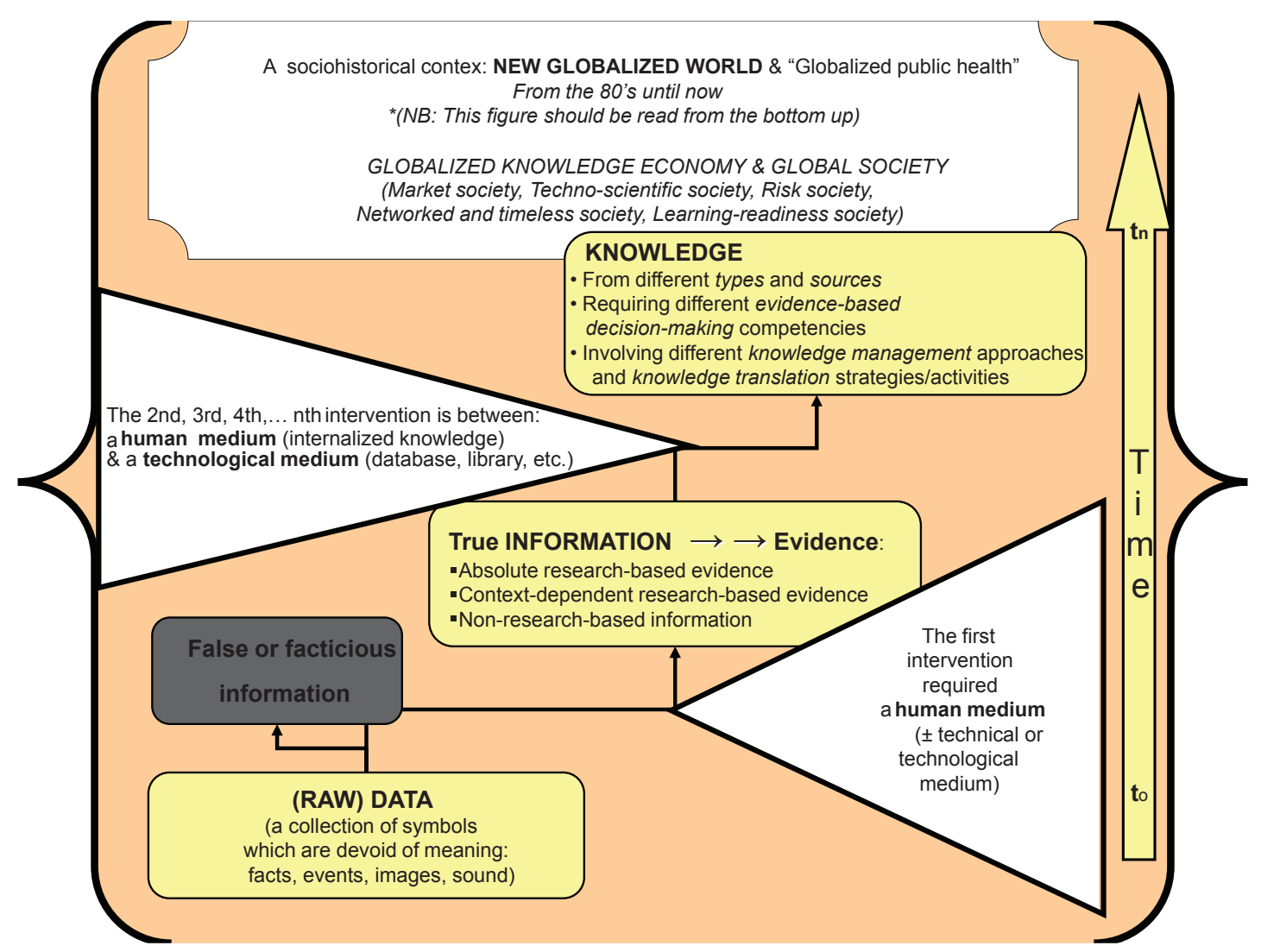

Figure $\mathbf{2}$ The "what" answer to evidence-based decision-making. 
(which can become context-dependent and research-based evidence), and nonresearch-based information. In health, these types of information and evidence are provided by different stakeholders (researchers and nonresearchers such as managers, clinicians, decision-makers, etc.) and through their respective working environments (work teams, peer groups, research teams, institutions, research centres, etc.). Following the intervention or the combined interventions of different media (human, technical and/or technological media), this evidence can be accessible for learning, and then internalized into knowledge and learned. Knowledge can be used through specific behavior and actions, sometimes it is transformed and, more often than not, it is capitalized: it becomes various types of knowledge (involving different knowledge-sharing strategies and requiring various evidencebased level-dependent competencies).

Third, there are different types of knowledge. Moreover, different knowledge typologies are defined and described in terms of purpose and use, type-conversion, structural features, properties, and conceptual levels. ${ }^{50}$ For the purpose and use of the PRO-ACTIVE program, the knowledge types are characterized as follows:

1. Declarative or propositional knowledge, also called factual knowledge, or "know-about" or "know-what" knowledge (eg, identify a specific health clinical procedure or practiceguidelines - as being efficient).

2. Contextual knowledge (also called background knowledge or "know-why" knowledge and associated with the kind of experience of the environment that is specifically required when new procedural knowledge is formulated and its rationale must be explained).

3. Procedural knowledge (or one's everyday know-how that may be difficult to express and based on factual and contextual knowledge).

4. Social knowledge (a type of meta-knowledge that, in comparison to individual knowledge, is closely related to collaboration/interaction and derived from group work or collective action).

Table I Evidence-based medicine and evidence-based health care management

\begin{tabular}{ll}
\hline $\begin{array}{l}\text { Specific level of the evidence-based } \\
\text { movement and level of evidence-based } \\
\text { competency to be developed }\end{array}$ & $\begin{array}{l}\text { Accessibility (I) and agentive } \\
\text { nature (2) }\end{array}$ \\
\hline $\begin{array}{l}\text { Evidence-based medicine (EBM), } \\
\text { Evidence-based clinical practice (EBP) }\end{array}$ & $\begin{array}{l}\text { I. Explicit knowledge (formalized, } \\
\text { conceptual and operational } \\
\text { objectives) }\end{array}$ \\
$\begin{array}{ll}\text { Essentially individual evidence-based decision-making } \\
\text { competency }\end{array}$ & $\begin{array}{l}\text { 2. Agentive nature = the individual* } \\
* \text { The clinician, the nurse } \\
\text { clinician, the health professional } \\
\text { who is in a "colloque singulier" } \\
\text { with the patient }\end{array}$
\end{tabular}

Evidence-based health care management, evidence-based decision-making* (Evidence-based public health, evidence-based health promotion, evidence-informed health policy, etc.) Essentially collective evidence-based decision-making competency

*Considered outside the clinical arena and the physician-patient relationship
I. Tacit (subjective, experiential, contextualized, informal) and implicit knowledge

2. Dominant agentive nature $=$ health organization* to which the manager, health or public health professional belongs

* = Quebec Health and Social Services System, the regional care systems coordinated by regional agencies, the local health and social services networks, health and social service centres, specialized hospitals, clinical programs
Types of knowledge

I. Reporting, propositional knowledge $=$ factual knowledge (know-how, know-about)

2. The contextual and procedural knowledge also brought to bear, but in a markedly reduced role (essentially in adapting research results, whereby the clinician and the researcher are in a synergistic relationship)

I. Contextual knowledge, background knowledge linked to experience in the practice setting (know-why)

2. Procedural knowledge, know-how without power of expression

3. Social knowledge, related to the inter-relational aspect and emerging from group work or collective action

4. Pragmatic knowledge, taking the form of efficient or promising practices, success stories, etc. 
5. Pragmatic knowledge (which is specific to one's meso level - group and/or organizational levels - and takes the form of best practices, promising projects, success stories, and business models or scenarios).

All these types of knowledge are critical to managing health care and developing knowledge-sharing platforms efficiently. However, depending on the specific level of the evidence-based movement (and in contrast to clinicians), pragmatic and social knowledge is more specifically the domain of health care managers (see Table 1).

Finally, depending on its accessibility, knowledge may be explicit, implicit or tacit. ${ }^{46,50}$ Explicit knowledge is available knowledge that is documented and takes the form of sources of formal knowledge (printed documents, electronic sources). It is codified and structured, and can be systematized. Conversely, tacit knowledge can be neither classified nor systematically indexed, and cannot be expressed outside of the actions of the person who possesses it. Tacit knowledge is therefore difficult to share, disseminate and learn, since it is stored by means of the replacement, from one generation to the next, of the individuals who carry it. Implicit knowledge, which is stored in human minds or organizations, is accessed by asking questions and engaging people in discussions, even though informal knowledge must first be identified and disseminated.

\section{"How?"}

In order to specifically address the issues implicit in the "What" and "How" of this paper, we have examined the relationships between the evidence-based decision-making movement (evidence-based medicine and evidence-based managerial practice), and the field of knowledge translation and knowledge translation research, with the different types of knowledge required; the accessibility of knowledge and the distinct agentive aspect at the specific decision-making level (is the level centered on the individual agent or the collective?); the type of professional training to be fostered; the storage medium specific to each of the two levels; and different issues of specific concern (see Table 1).
Type of professional learning

Essentially formal professional learning that occurs inside instituted educational structures (universities, schools, training centres, etc.) with renewed professional development approaches based on concepts of:

I. Self-learning

2. Learning technologies

3. Virtual coaching

4. Co-development groups

Informal professional learning

(= learning founded in the inter-relational dimensions of work - colleagues, superiors - and in the confrontation of professional uncertainties) operating outside instituted educational structures and for which the content is structured according to an action logic

Individual and organizational learning: I. Qualifying individual learning at the basis of group learning; learning from peers; organization in which members are constantly and proactively learning new things

2. Clear strategic intentions that foster learning
Storage medium + knowledge
translation tools
Specific issues

Technological medium

I. Codification tools: structured organization of evidence in databases (eg, systems of reference of explicit knowledge and their depositories, The Cochrane Library), data warehouses, inventories of best clinical practices

2. Dissemination tools: performance support systems (eg, knowledgebased CDSS)

Human medium (knowledge interiorized by the individual)

I. Codification tools: electronic data banks that codify tacit knowledge into explicit re-usable knowledge (eg, Success Stories Casebook) or systems of reference of tacit knowledge that has become explicit after having been documented from problems in the field and presented as efficient, exemplary or promising practices

2. Dissemination tools: Virtual or real practice communities, discussion forums, virtual or real coaching, mentoring.
Issue I: Create informational spaces (technological infrastructure for managing information: integrated electronic databases) Issue 2: Promote new approaches to professional development in instituted educational structures

Issue 3: As a professional, train oneself in EBM/EBP and integrate them in one's practice Issue 4: Beyond one's personal disciplinary affiliation, promote a trans-disciplinary approach to EBP in the health sciences Issue I:Accept and assume the TRIPLE challenge of:

a. Synergy between tacit and explicit knowledge b. Action learning (practice communities, forums, real or virtual coaching) and competency management (personal or group) c. Transforming personal knowledge into collective value added (creation/development of a group intelligence)

Issue 2: Developing integrated knowledge translation platforms to foster (explicit and tacit) knowledge

Issue 3: Development of actionable knowledge for decision-makers; development of knowledge-uptake activities among target audiences (managers and management staff) and evaluation of the impact of knowledgesharing activities at the meso and macro level 
With the 21 st century just begun, it is impossible to ignore the emerging field of knowledge translation and the evidence-based movement. The notion of evidence-based management of care and services - and the parallel notion of developing evidence-based competencies - is an effective response to the new relationship with knowledge promoted in global "learning-readiness" societies that foster performance in all their organizations, including health care organizations. Health care managers therefore feel they are subjected to many different beliefs, motivations, representations, and even affects that all vary but that, on the whole, are often favorable to learning: to the adoption of a knowledge-sharing attitude and the development of evidence-based decisionmaking competencies. However, as can be seen in Table 1, evidence-based health care management is not practised in isolation, like evidence-based medicine, nor does it originate from the "invariably prized aura" generated by systematized explicit knowledge.

\section{The PRO-ACTIVE research program}

The PRO-ACTIVE program (Participatory and Evaluative Research Program to Optimize Workplace Management: Application of Knowledge, Transfer of Expertise, Innovative Interventions, Training Transformational Leaders) is a participatory action research program focusing on the organization of care, services, and work (OCSW) through intense researcher/manager collaboration and a partnership. ${ }^{52}$ The PRO-ACTIVE program is funded by the Canadian Health Services Research Foundation and the Fonds de recherche en santé du Québec. As part of the PRO-ACTIVE research program, Canadian health care managers and researchers have decided to work together to identify avenues for optimizing human resources, processes and the psychological environment of health care professionals, in order to promote the provision of innovative, good quality care and services in the best working environment. PRO-ACTIVE, which is a sequential program of three complementary projects, is designed to generate knowledge, describe and evaluate innovative and inspiring management practices and to disseminate knowledge emerging from the research program. This new knowledge (different types of knowledge in their explicit and/or tacit dimension) will support managers of health care organizations in their evidence-based decisionmaking on reorganization of care, services and work, and promote transformational leadership as well as mentoring for managers of health care institutions and their partners in Canada's health care system.
The first project contributes, through formative and evaluative research (training of 300 managers), to development of a critical mass of evidence-based knowledge and skills in organization of care, services, and work (OCSW). The second project, through participative and evaluative action research including case studies of 30 organizational transformation projects, strives to identify innovative OCSW practices. Finally, the third project contributes through participative and evaluative research to the creation and evaluation of a mentoring network (cooperation and training of more than 30 expert mentors in OCSW), initiates implementation of a national network for mentoring and knowledge transfer in OCSW.

Within the PRO-ACTIVE program, knowledge and knowledge-sharing have both an individual and an organizational dimension. Different types of explicit and tacit knowledge are considered, and the interaction between two storage media (human mind and technological media) is promoted, the program also involving collaboration between the Canadian managers and academics. As portrayed in Table 1, knowledge take place at the micro, meso, and macro levels. Much of the elements gathered at the group level are data or information; information is then applied on experiences, learning groups, and training of managers to generate knowledge. This knowledge can remain tacit until it is called upon sharing with partners. Once gathered, information needs to be stored to allow further translation (transfer). Two strategies are common for capture and storage. The main strategy used by the program is the personalization strategy coupled with collaboration (partnership). This strategy focus on the dialogue and interaction between individuals, and to make the personalization strategies work, the program has developed a virtual community of people. Knowledge is shared not only in face-to-face but also by e-mail, over the phone and via videoconference.

\section{Conclusion}

Even though the goal of both evidence-based health care management and evidence-based medicine (evidence-based practice) is to improve the quality of health care and services, and even though the terms carry the same root ("evidence-based") and may be given the same acronym (EBM), it is important to draw a strong distinction between them. Evidence-based management fosters different types of knowledge that are tacit rather than explicit and that are directed at informal rather than professional learning. It is anchored in a learning-readiness process that is as much collective as it is personal, in the sense that managers' 
capabilities, aptitudes and qualities are inseparable from the work team's potential. In both cases, the goal is to produce organizational results. Evidence-based health care management also addresses very specific issues, encouraging the health care organization to make a strategic diagnosis of its knowledge management model, its knowledge-sharing activities and strategies, and then develop a sociotechnological and organizational infrastructure based on strategic orientations which crystallize both tacit and explicit knowledge at different levels. Then the "evidence-based" message will be a lever for the learning of different types of knowledge and for making such knowledge work together.

\section{Acknowledgments}

The author acknowledges with thanks the anonymous reviewers for their insights and comments towards the publication of this paper. Funding has been provided by the Canadian Health Services Research Foundation (CHSRF) and the Canadian Institutes of Health Research (CIHR). The views expressed herein do not necessarily reflect the views of either funding organization.

\section{References}

1. Lavis JN. Research, public policymaking, and knowledge-translation processes: Canadian efforts to build bridges. J Cont Educ Heath Prof. 2006;26(1):37-45.

2. Pabloz-Mendez A, Shademani R. Knowledge translation in global health. J Cont Educ Heath Prof. 2006;26(1):81-86.

3. Muir Gray JA. Evidence-Based Healthcare: How to make health policy and management decisions. London: Churchill Livingstone;1997.

4. Sackett DL, Rosenberg WMC, Gray JAM, Haynes RB, Richardson WS. Evidence based medicine: what it is and what it isn't. $\mathrm{Br}$ Med $\mathrm{J}$. 1996;312(7023):71-72.

5. Roberts AR, Yeager K. Systematic reviews of evidence-based studies and practice-based research: how to search for, develop, and use them. In: Roberts AR, Yeager K, editors. Evidence-Based Practice Manual: Research and outcome measures in health and human services. Oxford: Oxford University Press; 2004. p. 3-14.

6. Walshe K, Rundal TG. Evidence-based management: from theory to practice in healthcare. Milbank Q. 2001;79(3):429-457.

7. Dobrow MJ, Goel V, Upshur REG. Evidence-based health policy: context and utilisation. Soc Sci Med. 2004;58:207-217.

8. Boruch R, Petrosino A, Chalmers I. The Campbell Collaboration: A proposal for systematic, multinational and continuous reviews of evidence. London: School of Public Policy, University College London; 1999.

9. Bourgeois M-L. "Médecine apodictique" et médecine empirique: la place de l'EBM (evidence based medicine) en psychiatrie ["Apodictic medicine" and empirical medicine: the place of evidence-based medicine in psychiatry]. Encéphale. 2000;26(3):1-2.

10. Borenstein D. Evidence-based psychiatry. Psychiatr News. 2001 May 4, 2001;36(9):3.

11. Rushton JL. The burden of evidence. Br Med J. 2001;323:349.

12. Vermeire E. L'evidence based medicine apporte-t-elle également une réponse aux questions complexes? [Does evidence-based medicine answer to complex questions?] Minerva. 2005;4(2):18.

13. Williams DD, Garner J. The case against "the evidence": a different perspective on evidence-based medicine. Br J Psychiatry. 2002;180:8-12.
14. Cochrane A. Effectiveness and Efficiency: Random reflections on the health service. Leeds: Nuffield Provisional Hospitals Trust; 1972.

15. McSherry R, Pearce P, Tingle J. Clinical Governance: A guide to implementation for healthcare professionals. Oxford; Malden, MA: Blackwell Publishing; 2001.

16. Institute of Medicine. The National Round-table on Health Care Quality: Measuring the quality of care. Washington: Institute of Medicine; 1999.

17. Djulbegovic B, Morris L, Lyman GH. Evidentiary challenges to evidence-based medicine. J Eval Clin Pract. 2000;6:99-109.

18. Feinstein AR, Horwitz RI. Problems in the "evidence" of "evidencebased medicine." Am J Med. 1997;103:529-535.

19. Greenhalgh T. Narrative-based medicine: narrative based medicine in an evidence based world. Br Med J. 1999;318(7179):323-325.

20. Knottnerus A, Dinant GJ. Medicine based evidence, a prerequisite for evidence based. Br Med J. 1997;315(7116):1109-1110.

21. Maynard A. Evidence-based medicine: an incomplete method for informing treatment choices. Lancet. 1997;349(9045):126-128.

22. Tonelli MR. The philosophical limits of evidence-based medicine. Acad Med. 1998;73(12):1234-1240.

23. Buetow S, Kenealy T. Evidence-based medicine: the need for a new definition. J Eval Clin Pract. 2000;6:85-92.

24. Djulbegovic B, Morris L, Lyman G. Evidentiary challenges to evidencebased medicine. J Eval Clin Pract. 2000;6:99-109.

25. Miles A, Charlton B, Bentley P, Polychronis A, Grey J, Price N. New perspectives in the evidence-based healthcare debate. J Eval Clin Pract. 2000;6:77-84

26. Upshur RE. If not evidence, then what? Or does medicine really need a base? J Eval Clin Pract. 2002;8:113-119.

27. Rycroft-Malone J, Seers K, Titchen A, Harvey G, KitsonA, McCormack B. What counts as evidence in evidence-based practice? J Adv Nurs. 2003;51:71-81.

28. Guyatt G, Rennie D. The User's Guide to the Medical Literature: A manual for evidence-based clinical practice. Chicago, IL: AMA Publications; 2002.

29. Evidence-Based Medicine Working Group. Evidence-based medicine. A new approach to teaching the practice of medicine. JAMA. 1992;268(17):2420-2425.

30. Canadian Task Force on the Periodic Health Examination. The Canadian Guide to Clinical Preventive Health Care. Ottawa: Canada Communication Group; 1994.

31. US Preventive Services Task Force. Guide to Clinical Preventive Services, 2nd ed. Baltimore, MD: Williams and Wilkins; 1996.

32. Oxford Centre for Evidence-Based Medicine. Levels of evidence and grades of recommendations [cited 2008 Nov 17]. Available from: http://minerva.meniervation.com/cebm/doscs/levels.html.

33. Egger M, Davey Smith G. Potentials and promises. $\mathrm{Br}$ Med J. 1997;312:1371-1374.

34. Weiss CH. The many meanings of research utilization. Public Adm Rev. 1979;39:426-431.

35. Buchanan DR, Shaw S, Ford A, Singer M. 2003. Ethical issues in community based participatory research: Balancing rigorous research with community participation. Prog Community Health Partnersh. 2007;2(1):153-160.

36. Hanson D, Hanson J, Vardon P, et al. The injury iceberg: An ecological approach to planning sustainable community safety interventions. Health Promot J Austr. 2005;16(1):5-10.

37. Lavis JN, Robertson D, Woodside JM, McLeod CB, Abelson J. How can research organizations more effectively transfer research knowledge to decision makers? Milbank Q. 2003;81(2):221-248.

38. Health Canada. Vision and Strategy for Knowledge Management and IM/IT for Health Canada. Ottawa, ON: Health Canada; 1998.

39. Yalnizyan A. Getting Better Health Care: Lessons from (and for) Canada. Ottawa, ON: Canadian Centre for Policy Alternatives; 2006.

40. Lapaige V. La santé publique globalisée [Globalized Public Health]. Quebec City, QU: Presses de l'Université Laval; In press 2009.

41. Argyris C, Schon D. Organizational Learning. Reading, MA: Addison-Wesley; 1978.

42. Platon. Théétète. Paris: Flammarion; 1994. 
43. Locke J. An Essay Concerning Human Understanding (1690). Paris: Vrin; 2001.

44. Ballay JF. Tous managers du knowledge. Paris: Éditions d' Organisation; 2002.

45. Huber G. Organizational learning: The contributing processes and the literatures. Organizational Science. 1991;2(1):88-115.

46. Nonaka A. Dynamic theory of organizational knowledge creation. Organizational Science; 1994;5:14-37.

47. Brailer DJ. Management of knowledge in the modern health care delivery system. Jt Comm J Qual Improv. 1999;25:1.

48. Skyrme DJ. Capitalizing on Knowledge: From e-business to k-business. Oxford: Butterworth-Heinemann; 2001.
49. Davenport TH, Prusak L. Working Knowledge: How organizations manage what they know. Boston, MA: Harvard Business School Press; 1998.

50. Alavi M, Leidner, DE. Review: Knowledge management and knowledge management systems: Conceptual foundations and research issues. MIS Q. 2001;25(10):107-136.

51. Polanyi M. The Tacit Dimension. New York: Peter Smith Publisher Inc; 1967.

52. Viens C, Hains S, Lavoie-Tremblay M, et al. Participatory and Evaluative Research Program to Optimize Workplace Management: Application of knowledge, transfer of expertise, innovative interventions, training transformational leaders. CHSRF/REISS Competition; 2006-2010. 